\title{
Intrinsicality without Naturalness
}

\author{
D. GENE WITMER, WILLIAM BUTCHARD, AND KELLY TROGDON \\ University of Florida
}

\begin{abstract}
Rae Langton and David Lewis have proposed an account of "intrinsic property" that makes use of two notions: being independent of accompaniment and being natural. We find the appeal to the first of these promising; the second notion, however, we find mystifying. In this paper we argue that the appeal to naturalness is not acceptable and offer an alternative definition of intrinsicality. The alternative definition makes crucial use of a notion commonly used by philosophers, namely, the notion of one property being had in virtue of another property. We defend our account against three arguments for thinking that this "in virtue of" notion is unacceptable in this context. We also take a look at a variety of cases in which the definition might be applied and defend it against potential counterexamples. The upshot, we think, is a modest but adequate account of what we understand by "intrinsic property."
\end{abstract}

Some properties, we say, are intrinsic to the objects that have them, while others aren't. Several philosophical issues presume the distinction: we might debate over whether so-called secondary properties are intrinsic, whether mentality supervenes on intrinsic properties of the individual, whether certain goods are intrinsic goods, and so on.

Rae Langton and David Lewis (henceforth L\&L) have recently set out and defended an account of "intrinsic property" that makes use of two notions: being independent of accompaniment and being natural (1998). We find the appeal to the first of these promising; the second notion, however, we find mystifying. In this paper we argue that the appeal to naturalness is not acceptable ( $\$ 1)$ and offer an alternative definition of intrinsicality ( $\$ 2)$. The alternative definition makes crucial use of a notion commonly used by philosophers, namely, the notion of one property being had in virtue of another property. We defend our account against three arguments for thinking that this "in virtue of" notion is unacceptable in this context (\$3). We also take a look at a variety of cases in which the definition might be applied and defend it against potential counterexamples $(\$ 4)$. The upshot, we think, is a modest but adequate account of what we understand by "intrinsic property." 


\section{Against the appeal to naturalness}

\subsection{The Langton and Lewis approach}

The notion of an intrinsic property plainly has something to do with the notion of independence: an intrinsic property is one had in a way that is independent of how things are outside the individual in question. As is wellknown, however, this short slogan isn't enough to give us a noncircular definition. There is a trivial sense in which, for any property $\mathrm{P}$ you choose, whether an object $x$ has $P$ depends on what wholly distinct objects are like. For $\mathrm{x}$ to have $\mathrm{P}$ there had better not exist any wholly distinct individuals with the property of being unaccompanied by an individual with $P$. Of course, what we really want to say here is that a property $P$ is intrinsic just in case something's having $P$ doesn't depend on what intrinsic properties other individuals have.

How can we be rid of this circularity? If we continue to focus on the idea that intrinsic properties are independent of the way other things are, we may seem to be stuck having to find some way of distinguishing between those properties from which an intrinsic property must be independent and those from which it need not be independent. Instead of focusing on independence from the way other things are, however, we can focus on independence from whether other things are - that is, on independence from whether other things exist in the first place. While this line of thought is not one offered by L\&L, it is one we might well use to motivate the sort of account they propose. For the sort of account they propose seems inspired by just this thought: a property is intrinsic just in case whether or not an individual has it is independent of the existence of other individuals.

L\&L make this notion precise by defining "independent of accompaniment" as follows. First, let an accompanied individual be one which coexists with some contingent individual wholly distinct from it and a lonely individual one that is not accompanied. We can then say that a property $\mathrm{P}$ is independent of accompaniment just in case each of the following four situations is possible: a lonely individual has P; a lonely individual lacks P; an accompanied individual has $P$; and an accompanied individual lacks $P$.

Since independence of accompaniment seems motivated by the general idea of independence central to intrinsicality, it's tempting simply to identify the two in what we call the Simple Independence Definition:

A property $\mathrm{P}$ is intrinsic iff $\mathrm{P}$ is independent of accompaniment.

As appealing as the Simple Independence Definition is, however, it clearly won't do. Consider this "disjunctive" property: being cubical and lonely or non-cubical and accompanied. This property is independent of accompaniment: it's possible for a lonely individual to have it (by being a cube that is lonely), for a lonely individual to lack it (by being a non-cube that is lonely), 
for an accompanied individual to have it (by being a non-cube that is accompanied), and for an accompanied individual to lack it (by being a cube that is accompanied). Yet the property of being cubical and lonely or non-cubical and accompanied is intuitively not intrinsic. Any number of examples of this sort can be cooked up. Think of this as the "disjunction problem."

To solve the disjunction problem, L\&L appeal to the notion of a natural property in the following way. They define "disjunctive property" in either of two ways, depending on whether naturalness is a matter of degree. If it is not a matter of degree, then a property is disjunctive iff it can be expressed as a disjunction such that the disjuncts are natural properties. If naturalness is a matter of degree, then a property is disjunctive iff it can be expressed as a disjunction such that the disjuncts are more natural than the disjunction itself.

Given an account of "disjunctive," $L \& L$ then define intrinsicality in two steps. First, they define a "basic" intrinsic property thus:

Property $P$ is a basic intrinsic property iff:

(1) $\mathrm{P}$ is independent of accompaniment; and

(2) $\mathrm{P}$ is neither a disjunction nor a negation of a disjunction.

Finally, an intrinsic property generally is defined:

Property $P$ is an intrinsic property iff:

$\mathrm{P}$ is either a basic intrinsic property or $\mathrm{P}$ is definable using basic intrinsic properties and truth-functional compounds thereof. ${ }^{1}$

Call this the Naturalness Definition.

\subsection{The high cost of naturalness}

It's on all hands agreed that an appeal to "naturalness" is a theoretical cost that should be avoided if possible. The question, it seems, is whether it can be avoided. In response to a critical paper by Marshall and Parsons (2001), Lewis says:

Dan Marshall and Josh Parsons have misgivings about the very idea of judging comparative naturalness. Well, anyone should prefer to do without these judgements, since they rest either on contentious ontology or on a primitive distinction. But such judgements are in fact made, often with confidence, and if you had to do without them, inability to define "disjunctive property" or "intrinsic" would be the least of your worries. (2001: 381-2)

1 In a later paper, "Redefining Intrinsic" (2001), Lewis advances a different account which boasts, he says, the advantage of simplicity. Since it too makes crucial use of the notion of naturalness, we reject it for the same reasons we reject the 1998 account. 
We think that this way of talking about the costs involved is a bit misleading. It is not as if we can appeal to naturalness but are merely hesitant to do so because of the commitments it incurs. Rather, we don't know how to evaluate naturalness claims in the first place. The problem here is not a matter of having a concept and assessing the costs and benefits of using it. Rather, the problem is a matter of having no clear concept the costs and benefits of which can even begin to be assessed.

It may be that our failure to grasp the relevant concept of naturalness is an artifact of L\&L's official neutrality on what naturalness comes to. We note that in the original paper they try to make the appeal to naturalness as harmless as possible, pointing out that there are several different ways of thinking about the distinction:

Some of us will help ourselves to some sort of primitive notion of naturalness of properties. Others will accept an ontology of sparse universals, or of sparse tropes, that has a built-in distinction between natural properties and other properties. Still others will wish to characterize the natural properties as those that play some interesting special role in our thinking-but for our present purposes, even this vegetarian metaphysics will suffice. One way or another, most of us will be prepared to grant such a distinction. (1998: 119-120)

A thorough investigation of the Naturalness Definition would require us to examine it under various interpretations corresponding to the various ways one might cash out "natural property." While Lewis himself has acknowledged that some ways of understanding "natural property" have the result that the Naturalness Definition of intrinsicality fails (2001: 382), there may be other ways of understanding it which will result in an acceptable definition. ${ }^{2}$

But we doubt it. While we can't provide an exhaustive survey of ways one might provide an account of naturalness, we'll take a quick look at the two approaches we find most plausible. The first is one Lewis refers to approvingly as a "vegetarian" conception which he thinks will serve us in defining intrinsicality. According to this account, a property is natural to the extent that it plays a "central and fundamental classificatory role within regimented physics (or perhaps within future unified science)" (2001: 382, n. 5). ${ }^{3}$

We find the Naturalness Definition understood given this conception of naturalness highly dubious. Since $L \& L$ define "intrinsic" in terms of naturalness, our judgements about which properties are intrinsic end up, on this account of naturalness, being defeasible on empirical grounds in a way that seems plainly inappropriate. To make the point vivid, consider an example of

2 The account that renders their definition inadequate is that which construes natural properties as those familiar to common sense. Unfortunately, this is precisely the one account of "natural property" that makes it relatively easy to classify properties as natural or not.

3 Lewis here refers to an account developed in Barry Taylor's 1993 paper "On Natural Properties in Metaphysics." We take it that by "vegetarian" Lewis means an account that is minimally committed to controversial metaphysical machinery. 
a "disjunctive" property akin to that which motivates L\&L to appeal to naturalness-say, being either a lonely solid or an accompanied liquid. This property meets the first condition on being a "basic intrinsic property," as it is independent of accompaniment (we could have a lonely solid, a lonely nonsolid, an accompanied liquid, or an accompanied non-liquid). If it is also natural, then, it is a basic intrinsic property on L\&L's definition. ${ }^{4}$ Our question now is: given this "vegetarian" conception of naturalness, could this property-being a lonely solid or an accompanied liquid-turn out to be natural? Answer: if and only if it could turn out that this property plays a central and fundamental classificatory role in physics or some successor, unifying theory. We can see no way to rule out such a role. Suppose the world is in fact much stranger than we thought, and the best way to produce a simple, systematic and informative account of the laws of nature is to allow this property in as one of the fundamental categories. In that case, we'd have to say that the property of being a lonely solid or an accompanied liquid is actually intrinsic. We find this an absurd consequence. While we don't want to say that every property can be a priori classified as intrinsic or not, we do want to say that the scenario just presented should not give us reason to reclassify the property of being a lonely solid or accompanied liquid as being, after all, intrinsic. ${ }^{5}$ The "vegetarian" approach to naturalness, then, seems unlikely to give us a plausible account of intrinsicality.

The other approach to naturalness, more ontologically committed, is that which Lewis spotlights in his "New Work for a Theory of Universals":

Because properties are so abundant, they are undiscriminating. Any two things share infinitely many properties, and fail to share infinitely many others. That is so whether the two things are perfect duplicates or utterly dissimilar. Thus properties do nothing to capture facts of resemblance. That is work more suited to the sparse universals... Properties carve reality at the

4 We're assuming in the main text that naturalness is not a matter of degree, since that assumption fits most easily into the conception of naturalness on the table. If we want to make room for naturalness being a matter of degree on this "vegetarian" conception, the idea would presumably be that $F$ is more natural than $G$ just in case the role of $F$ in physical theory-or whatever theory is ultimately at issue here-is more fundamental than the role of $\mathrm{G}$ in that theory. Our following remarks about the property of being either a lonely solid or an accompanied liquid can then be adjusted accordingly so that the unlikely scenario we're imagining is one in which the property of being either a lonely solid or an accompanied liquid plays a more fundamental role in the relevant theory than either the property of being a lonely solid or the property of being an accompanied liquid. Why are we so confident of this? We think anyone will agree with this conclusion if he grants something else that seems to us obvious, namely, that the property of being a lonely solid or accompanied liquid is capable of being instantiated by virtue of one of its disjuncts' being instantiated. Something could have this property by virtue of being a lonely solid. But being a lonely solid is not even independent of accompaniment, much less intrinsic. Of course, our own proposed account of intrinsicality predicts that this is a good reason to deny that this property is intrinsic (see \$2), but we think the argument for counting it as non-intrinsic should be persuasive even to those who have not adopted our definition. 
joints-and everywhere else as well. If it's distinctions we want, too much structure is no better than none.

It would be otherwise if we had not only the countless throng of properties but also an elite minority of special properties. Call these the natural properties. If we had properties and universals both, the universals could serve to pick out the natural properties. (1983: 13).

This way of making sense of "natural" may be fine as it stands, but if we look to it as a way of making sense of "natural" that can in turn be used to illuminate "intrinsic," we think the approach futile. It is indeed hard to imagine a property being intrinsic which doesn't make for resemblance. But this, we take it, is for a rather tedious reason. The "resemblance" that Lewis invokes is understood in the first place as resemblance with respect to intrinsic properties. If we are told that resemblance here means resemblance period, not resemblance with respect to some kind of property, then we insist that to talk of such resemblance is just a category mistake. We maintain both that (i) any talk of resemblance must be understood in terms of resemblance with respect to some class of properties; and (ii) the ordinary talk of resemblance which so impresses Lewis can be understood as implicitly restricted to resemblance with respect to intrinsic properties.

The upshot, we think, is this. If we want to clarify a notion which is a relatively ordinary one, presumably the notions we use to provide that clarification ought to be in better shape than the one to be clarified. But that principle is turned on its head in the case of the Naturalness Definition. Which of the two concepts-that of intrinsicality and that of naturalness-is better understood? We think it is no contest. If we insist on a use for the notion of "natural property," it seems more likely that naturalness is to be analyzed in terms of intrinsicality, not vice versa.

\section{The Strong Independence Definition}

\subsection{Introducing the alternative}

We want now to show how we can define intrinsicality without such appeal. Let's backtrack to the point at which we saw the inadequacy of the Simple Independence Definition:

A property $P$ is intrinsic iff $P$ is independent of accompaniment.

As we saw, the problem with the Simple Independence Definition is that it is too weak: it counts as intrinsic several properties that intuitively aren't intrinsic, such as the property of being cubical and lonely or non-cubical and accompanied. We need some way of ruling out such properties.

Let's think about why, intuitively, we reject that property as non-intrinsic. Suppose some individual a has this property; suppose, in fact, that a has this property because it is cubical and lonely. In that case, its having this property is due to its being cubical (presumably an intrinsic property) and its 
being lonely (which is clearly not an intrinsic property). So its having the disjunctive property in question is dependent on its non-intrinsic properties. It is, we maintain, the fact that it is so immediately obvious that an individual could have the disjunctive property in virtue of its having certain plainly nonintrinsic properties that drives us to classify the disjunctive property as nonintrinsic itself.

In general, if a property $P$ can be possessed by an individual in virtue of that individual having some property $Q$, where $Q$ is not itself intrinsic, then $P$ is not intrinsic. Equivalently, it's a necessary condition on P's being intrinsic that, for any $Q$ such that $P$ is had by virtue of $Q, Q$ is intrinsic as well.

Does this get us anywhere? We've not eliminated the use of "intrinsic" yet. But perhaps we can do so if we return to the requirement that an intrinsic property must be independent of accompaniment. Suppose we substitute "independent of accompaniment" for "intrinsic" in the above necessary condition and then offer that as a condition which, when added to the Simple Independence Definition, provides us with a condition both necessary and sufficient:

Property $\mathrm{P}$ is intrinsic iff (i) $\mathrm{P}$ is independent of accompaniment, and (ii) for every $Q$ such that $P$ is had by virtue of $Q, Q$ is independent of accompaniment.

Unfortunately, the second-clause is ill-formed as it stands. Whether $P$ is had by virtue of some given $Q$ depends on the particular instance of $P$. Consider our example of a disjunctive property again, the property of being cubical and lonely or non-cubical and accompanied. In the example we imagined, an individual had this property by virtue of being cubical and lonely. It's also possible, of course, for an individual to have this property by virtue of being, say, triangular and accompanied. It makes no sense to say that $P$ is had by virtue of $\mathrm{Q}$, simpliciter; we must relativize the question to individuals and ask, in the case of any individual $x$ with $P$, what is it about $x$ that makes it the case that $x$ has the target property $P$ ?

How, then, to define "intrinsic property"? We suggest a two stage process: first, define what it is for an individual to have a property in an intrinsic fashion; second, define a property as intrinsic just in case any possible instance of it is one that is had in an intrinsic fashion. To have a property in an intrinsic fashion is simply to have a property that is independent of accompaniment and which, if had by virtue of any more fundamental properties, is had by virtue of properties all of which are themselves independent of accompaniment. In this way, independence of accompaniment is revealed to be at the heart of the notion. 
Our proposal modifies the Simple Independence Definition by taking into account the fact that some properties are instantiated by virtue of the instantiation of other properties. Since the new definition is still very much in the same spirit as the original while being stronger, we'll call this the Strong Independence Definition:

Property $\mathrm{P}$ is intrinsic iff, for any possible individual $\mathrm{x}$, if $\mathrm{x}$ has $\mathrm{P}$, $\mathrm{x}$ has $\mathrm{P}$ in an intrinsic fashion.

$X$ has $P$ in an intrinsic fashion iff (i) $P$ is independent of accompaniment and (ii) for any property $Q$, if $x$ has $P$ in virtue of having $Q$, $Q$ is also independent of accompaniment.

The Strong Independence Definition handles easily the example that raised the disjunction problem: being cubical and lonely or non-cubical and accompanied. That property can be had in a non-intrinsic fashion, since it might be had by virtue of being cubical and lonely, which property is not independent of accompaniment. Since it can be had in a non-intrinsic fashion, it is not classified as itself intrinsic. In light of this, the definition seems to us both simple and intuitively plausible. We will, of course, discuss several more examples later (see §4).

\subsection{Two features of our definition}

Before moving on to consider objections, we want to note two features of the definition. First, our definition requires us to make use of a relativized notion of having a property in an intrinsic fashion. We suspect that any attempt to do without such a notion is futile and that premature dismissal of such a notion may serve only to block efforts at explicating intrinsicality. We were surprised to see the notion dismissed as simply a non-starter in a recent paper. In his commentary on the Naturalness Definition, John Hawthorne considers the property of attending to someone or other (the "existential derivative" of the relation of attending) and says, as an aside:

I assume we do not wish to relativize matters so that we count the existential derivative an intrinsic property of Jones (who is attending to his leg) but not of me (who is attending to the table). (Hawthorne 2001: 400, n. 1)

Pace Hawthorne, we think this is exactly what should be done. Indeed, others who have tackled the question of intrinsicality have acknowledged the need for such a relativization. J. M. Dunn (1990: 183), I. L. Humberstone (1996: 206), and Robert Francescotti (1999: 590) have each employed a relativized notion. Hawthorne's own example we think makes the point. When Jones attends to his leg, he has the target property in an intrinsic fashion; but when I attend to the table, I have it in a non-intrinsic fashion. But perhaps 
another example will make the need for this relativization more apparent. Consider the property of being triangular or being noncubical and accompanied. This property is independent of accompaniment. (Four possible cases: a lonely triangle; a lonely cube; an accompanied triangle; and an accompanied cube.) And it can be had both in an intrinsic fashion and in a non-intrinsic fashion. Suppose $a$ is triangular. In that case, it seems that a has the property of being triangular or noncubical and accompanied by virtue of being triangular-a property we presume is intrinsic. There, it seems a has the target property in an intrinsic fashion; we need nothing but the way a intrinsically is to explain why it has the target property. If, on the other hand, we consider an individual $b$ which has the target property by virtue of being a hexagonal accompanied object, we are forced to say that $\mathbf{b}$ has the property in a nonintrinsic fashion.

The second feature of the Strong Independence Definition we should note is that it implies (given certain plausible assumptions) that two properties which are necessarily coextensive can yet differ in whether they are intrinsic. Let $P$ be any property counted as intrinsic by our definition; now consider the property of having $P$ and being accompanied or having $P$ and being lonely. This latter property-let us abbreviate it as "(P\&A)v(P\&L)"-is necessarily coextensive with $P$, yet, plausibly, it will not be counted as intrinsic by our definition, for it could be instantiated by virtue of something's having $P$ and being lonely, which property is not independent of accompaniment. Is this an unhappy consequence of our definition?

Here is one reason it may be unwelcome. Suppose we accept the coarsegrained view of properties according to which necessarily coextensive properties are identical; in that case, the Strong Independence Definition implies that being intrinsic cannot be counted as a genuine feature of properties: properties are only intrinsic or not under a given description, or when conceived of in a certain way. Such an implication may suggest that debates about whether a given property is intrinsic should not assume much ontological weight. If we want to avoid this result, we could, of course, reject the coarse-grained view of properties. Another option is to modify the definition of "intrinsic" so that if any property P counts as intrinsic by the present definition, then any $Q$ which is necessarily coextensive with $P$ thereby counts as intrinsic as well. We do not here take a stand on which option is preferable.

We do, though, want to stress that the Naturalness Definition faces the same consequence. Suppose $\mathrm{P}$ is a basic intrinsic property by that definition; how then does that definition classify the property $(P \& A) v(P \& L)$ ? It looks at first as if it implies that while $P$ is intrinsic, (P\&A)v(P\&L) is not. Note that the expression "having $\mathrm{P}$ and being accompanied or having $\mathrm{P}$ and being lonely" is not a definition built up in a truth-functional way out of nothing but names for basic intrinsic properties. So at first it looks as if 
(P\&A) $v(P \& L)$ is counted by the Naturalness Definition as not intrinsic. But there is a way of understanding that definition which counts it as intrinsic anyway. Suppose one likes the coarse-grained view of properties; one might then say that for any properties $F$ and G, F is "definable" as $G$ just in case $F$ and $G$ are necessarily coextensive. In that case, the Naturalness Definition does count $(\mathrm{P} \& \mathrm{~A}) \mathrm{v}(\mathrm{P} \& \mathrm{~L})$ as intrinsic, since $(\mathrm{P} \& \mathrm{~A}) \mathrm{v}(\mathrm{P} \& \mathrm{~L})$ is definable simply as $\mathrm{P}$ itself-and thereby is definable as a truth-functional compound of basic intrinsic properties. In the same way, if we so wished, we could define an intrinsic property as one which is definable as one which counts as intrinsic under the original definition. On this point, then, the Naturalness Definition and Strong Independence Definition are on a par. .

\section{Three objections to our account}

\subsection{Our use of "in virtue of"}

The most likely objections to our account, we expect, will center on the fact that our definition makes crucial use of the phrase "in virtue of." Indeed, such an objection is almost anticipated in the following passage from Lewis' "Redefining Intrinsic":

In general, something has an intrinsic property solely in virtue of how that thing itself is; it has a purely extrinsic property solely in virtue of how accompanying things, and its external relations to these accompanying things, are; and it has a non-intrinsic but not purely extrinsic property in virtue partly of the former and partly of the latter. (If we had a clear enough understanding of 'solely in virtue of', we would need no further definition of 'intrinsic'.) (2001: 384).

The claim here seems to be that the "in virtue of" locution is both essential to understanding intrinsicality and itself in need of clarification. We can distinguish three objections that seem to be suggested here. The obscurity objection complains that the "in virtue of" notion is just as unclear as the notion of a natural property. Even if that notion is reasonably clear, one might complain that an appeal to it would render a definition of intrinsicality circular: this is the circularity objection. Finally, even if neither of the first two objections succeed, one might wonder about the utility of a definition of "intrinsic" that requires us to appeal to "in virtue of": this more generic worry is the utility objection.

\subsection{The obscurity objection}

If our understanding of "in virtue of" is as poor as our understanding of "natural property," then, plainly, the Strong Independence Definition has no advantage over the Naturalness Definition. Is our understanding of "in virtue of" indeed that poor?

When we ask about the quality of our understanding of a notion, we should distinguish between two very different questions. We might be asking 
about our ability to produce an explicit theory about the notion in question-a theory about its logical features, its relation to other notions, perhaps even a definition of it. We might be asking a different question, however: we might be asking about the degree to which we actually have a facility with the concept-how confidently we use it, whether we can reach agreement on its proper use in a reasonable range of cases, whether our judgements involving it are stable and not subject to inexplicable shifts, and so on.

While we freely admit that we lack anything like a definition of "in virtue of", we think that when it comes to the matter of facility with the notion, our grasp of the notion is relatively secure. Further, we think that we can say something by way of theoretical description of the notion. The same, we think, cannot be said of the notion of a natural property-or, at least, of the deliberately "neutral" notion of "natural property" that L\&L employ.

Let us focus on the point about facility. Philosophers and non-philosophers alike make use of "in virtue of" on a regular basis; there is, further, robust agreement on its proper use in philosophically uncontroversial contexts. It is especially easy to find ordinary language examples of "in virtue of" when we look to talk about rights, responsibilities and powers one acquires from the societal roles one plays; for instance, one has the right to vote in virtue of being an adult citizen, the responsibility to care for one's children in virtue of being a parent, and the power to expel a student from school in virtue of being a high school principal. One good way to appreciate how commonly used the notion is in ordinary thought is to bear in mind that besides "in/by virtue of" there are other expressions that do the same work. "Makes" is often used in this capacity; indeed, we could hardly ask for a better example of a thoroughly unexceptional use of the notion in ordinary language than this: "what makes you so smart?"

It's not just "in virtue of" and "makes," however, that can be used to get at the relevant notion. If we venture a bit into more artificial jargon we can capture it in a variety of ways. Suppose, for instance, that we have agreed to the following claim:

Jack is sick in virtue of having a cold.

We are then likely to agree with the following claims as well:

Jack has a cold and thereby is sick.

Jack's having a cold counts as his being sick.

Jack's having a cold makes true the claim that he is sick.

That Jack is sick is nothing over and above the fact that he has a cold. 
We put forward these similar sounding claims not to suggest that they are synonymous with the original; we expect, rather, that there is a single core notion by reference to which these various expressions can all be defined. The point is twofold. First, we take these expressions to indicate that there is a relatively well-understood notion that shows itself in a variety of expressions. Second, the variety can be used to help pinpoint which notion, exactly, is at issue. They might not offer an explanatory reduction of the notion in any sense; but they can help ensure that we are successfully communicating. If we are unsure how to evaluate a sentence using "in virtue of," we can shift focus to the corresponding sentence using a different locution. If, for example, we're not sure we understand the question "is a person dead in virtue of his or her having no heartbeat?" we can clarify the question by considering a closely associated question: "is having no heartbeat the sort of thing that makes it true that a person is dead?"

This said, we confess that, of course, we would like to have a theory of the "in virtue of" notion. But we stress that lacking such a theory is a far cry from failing to have enough of a grip on the notion to evaluate a wide variety of claims made using it. Compare: we may lack a good theory about the analysis of " $S$ knows that $p$," but for many cases we are hardly at sea when asked to evaluate whether a particular person knows a particular proposition (bracketing skeptical worries).

With "natural property," however, we find ourselves very much at sea. To put the point in frankly phenomenological terms: when asked to evaluate whether a given property is natural or more natural than another, we find ourselves forced to put the question in other terms first, relying on what others have given as ways of explicating "natural." We find ourselves wondering: Are you asking if it's intrinsic? If it's likely to appear in a law of nature? If it's among the properties in terms of which all other properties can be defined ${ }^{6}$ By contrast, we don't find ourselves automatically grasping for such reformulations when asking whether one thing is true in virtue of another.

We don't think we're alone in this. Nearly every philosopher, we venture to say, makes use of the "in virtue of" notion on a regular basis; this is not the case with the notion of a natural property. In light of this we think it's clear that the philosophical community as a whole has a better grasp on "in virtue of" claims than it does on "naturalness" claims.

\subsection{The circularity objection}

Even if the "in virtue of" notion is one with which we have a reasonably good facility, the notion had better not be parasitic on the target notion of intrinsicality. We can distinguish two sorts of circularity we might worry about. Let us say that a definition is directly circular just in case one of the

6 Are we supposed to presume that all of these features necessarily go together? 
notions used in the definition is equivalent to the notion being defined, even if it appears under a different name. A weaker charge can be defined as well. Let us say that a definition is indirectly circular just in case some notion used in the definition is such that anyone who grasps it can only do so because she has already grasped the notion being defined.

It is quite obvious that the Strong Independence Definition is not directly circular. No one could maintain that "in virtue of" and "intrinsic" are equivalent-to suppose they are would be to commit a particularly gross category mistake. Might it, however, be indirectly circular?

We find this charge entirely implausible. It is not as if the only place the "in virtue of" notion shows up in philosophy is in discussion of intrinsicality. Practically everywhere in philosophy the notion is apt to turn up: What makes an action right or wrong? By virtue of what is this individual a conscious being? What makes a counterfactual true? By virtue of what does this person count as understanding English? And so on. The central use of the notion suggests that other, less central notions are understood by means of understanding $i t$, not vice versa. The Strong Independence Definition seems to us neither objectionably obscure nor circular in either of these senses.

\subsection{The utility objection}

Even if neither the obscurity or circularity objection succeeds, one might still complain that the Strong Independence Definition does not live up to expectations given its use of the "in virtue of" locution. The complaint, more precisely, is this: Since we bothered ourselves about trying to come up with a definition of "intrinsic" in the first place, we must have in mind some restricted set of notions in terms of which we want to define "intrinsic"-such as modal concepts, part-whole relations, and so on. But (the objection continues) it's likely that this restricted set does not include the "in virtue of" notion. One might put the question this way: What is the use of the definition if it does not aspire to greater heights than this?

To assess this objection we need to face squarely the question as to why we should want an analysis of "intrinsic" in the first place. Once we have a good answer to that question we will be well placed to see if the motivation is happily served by our Strong Independence Definition.

Here's one reason one might want an analysis of "intrinsic": One might endorse some sweeping thesis about the definability of a wide range of concepts in terms of some privileged set. If, for instance, one comes to the table already convinced that all metaphysical concepts are definable in terms of linguistic concepts in the fashion of Carnap, one might be motivated to define " $\mathrm{P}$ is an intrinsic property" as a statement about certain predicates. So far as we can tell, no such sweeping thesis is behind the efforts that have been made to define "intrinsic." 
Another motivation, of course, is simply that it would be nice to simplify matters; if we can provide a definition of "intrinsic" in other terms, that would reduce the number of primitives. This we suspect is behind many efforts to define "intrinsic." But it obviously places no interesting constraints on acceptable definitions in the absence of a larger commitment to eliminating other primitives. If one is pursuing a simplification project and has already decided that "in virtue of" is not to be one of the primitives, then of course one has a motivation to avoid our definition; but this just pushes the question back as to why we should suppose that that notion should not be one of the primitives.

The one clear and compelling motivation we can find for pursuing a definition of "intrinsic" is that there is a puzzle about the relation between our actual notion of intrinsicality and the usual ways in which we explain what notion we have in mind. Consider how philosophers typically explain what is meant by an "intrinsic property." An intrinsic property, it is said, is one which "things have in virtue of the way they themselves are" (Lewis 1986: 61). It is a property "that is internal in the sense that whether an object has it depends entirely upon what the object is like in itself" (Francescotti 1999: 590). A property is intrinsic "just in case a thing's having it (at a time) depends only on what that thing is like (at that time), and not on what any wholly distinct contingent object (or wholly distinct time) is like" (Vallentyne 1997: 209). Call these the "orienting characterizations" of intrinsicality.

These orienting characterizations manage to direct our attention to the notion of an intrinsic property; they result in our being able to agree in classifying a wide range of properties as intrinsic or not. There is a question, however, about how they do so. On one straightforward way of interpreting them they fail to capture our actual notion of intrinsicality. To get an interpretation of them that does justice to that notion, we need to interpret them in a blatantly circular way.

There are fundamentally two themes running through the usual orienting characterizations. One is that an intrinsic property is one that doesn't depend on the way other things are. The problem with this characterization is wellknown; every property $P$ is trivially dependent on how other things are in the sense that other things had better not have the property of being unaccompanied by something with $\mathrm{P}$. If, however, we interpret the characterization as saying that an intrinsic property is one that doesn't depend on the way other things are intrinsically, of course, the problem does not arise. But then it is puzzling how the use of this orienting characterization succeeds in directing our attention to the notion of intrinsicality.

The other theme to be found in the orienting characterizations is that an intrinsic property is one had solely by virtue of the way the object itself is. As before, this admits of a straightforward interpretation which gets us the 
wrong result. Consider an individual, Olive, who is standing by an aquarium containing an octopus. She has the property of being within three yards of an octopus. Does she have this property by virtue of the way she herself is? $\mathrm{O}$ course she does; she has it by virtue of being located in that environmer: with such and such an arrangement of objects, including the relevant octopus. And all of that is, of course, the way she is. To stress that it's a property had by virtue of the way she herself is doesn't help: it's not as if we're making a mistake in attributing the property to her as opposed to something else. What would help, of course, is if we understood the claim as saying that an intrinsic property is one which is had by virtue of the intrinsic way the object itself is. But again, this raises a puzzle: if the characterization only fits our notion when understood in this circular fashion, how does it help?

This fact about the usual orienting characterizations provides us with a motivation for defining "intrinsic." We want a characterization of our notion that is not only accurate but which allows us to explain how the usual orienting characterizations manage to convey the notion we evidently have.

On this account of the motivation, there is no specified set of privileged notions in terms of which intrinsicality must be explained; hence, it cannot be complained that our use of "in virtue of" fails to be among that set. On the other hand, one might wonder whether our account renders intelligible the connection between the orienting characterizations and our notion of intrinsicality.

Here, we think, the strength of our account is manifest. The definition suggests a simple and plausible story about how the usual orienting characterizations succeed in focusing attention on the notion. When it is said that an intrinsic property is one that is had by virtue of the way the object itself is, we are directed to look at the bottom-level properties that ground the others; when it is said that an intrinsic property is one that is independent of the way other things are, we are directed to look to see if the grounding properties are independent of accompaniment. Together, the two themes running through the orienting characterizations direct us to look at more basic properties to see if any fail to be independent of accompaniment. In other words, they direct our attention to that which, on our account, makes a property intrinsic or not-whether it is always grounded in properties that are independent of accompaniment.

\section{Applying the definition}

\subsection{Can the definition be fairly assessed?}

If we are to defend the Strong Independence Definition, we need to be able to apply it to a variety of cases and examine the results. It is crucial, of course, that our application not be ad hoc; the judgement that a given property is or is not instantiated by virtue of another had better not be tailored simply to 
defend our definition. Since we lack a definition of "in virtue of," intuitive judgements will play a large role in assessing our account. We don't think we are entirely deprived of theoretical guidance, however; there may be some limited principles that can help guide such judgements.

One such principle concerns sufficiency. We think it intuitively clear that, if we list all the properties in virtue of which a particular individual has some other property $P$, then the joint instantiation of those properties is in general sufficient for the instantiation of $P$. Call this the Joint Sufficiency Principle. More precisely:

(JSP) For any individual $x$ and property $P$, if there is a non-empty set of properties $S$ such that $Q$ is a member of $S$ iff $x$ has $P$ in virtue of having $Q$, then: for any individual $y$, the joint instantiation of every member of $S$ by y necessitates the instantiation of $P$ by $y$.

We stress that the principle is not a biconditional. If we have a true "in virtue of" claim, then we will have such a jointly sufficient set; but not every set of jointly sufficient properties will yield an "in virtue of" claim. An "in virtue of" claim carries some explanatory force, but simple entailment is not enough to guarantee explanatory force. It's important for our account of intrinsicality that this be acknowledged; the following example should make the point vivid. Suppose an individual $\mathbf{a}$ is an accompanied sphere and has, therefore, this property:

(E1) Being a sphere

Now a also has these two properties:

(Q1) Being accompanied

(Q2) Being either cubical and lonely or spherical and accompanied

The joint instantiation of $\mathrm{Q} 1$ and $\mathrm{Q} 2$ by any individual will necessitate the instantiation of E1. If, however, we were to say that a has E1 by virtue of having Q1, we'd have to say that a does not have E1 in an intrinsic fashion, since Q1 is (obviously) not independent of accompaniment. We take it, however, that there is no intuitive pull whatsoever towards saying that $\mathbf{a}$ is spherical by virtue of being accompanied and being either cubical and lonely or spherical and accompanied. We admit, then, that the Joint Sufficiency Principle hardly allows us to eliminate the appeal to intuition.

The principle can nonetheless be of some use. If we advance a particular "in virtue of" claim, we can test it for conformity to the principle; we can see if there exists an appropriate set of jointly sufficient properties. In doing so, 
we can also be made to notice other grounding properties that may be relevant to our assessment of the Strong Independence Definition.

With those points in mind, let us illustrate the utility of our definition with a few examples. Here are two cases taken from the recent symposium in Philosophy and Phenomenological Research on the Naturalness Definition:

(E2) Being the only round thing (Weatherson 2001: 367)

(E3) Being one of at most 17 cubes (Weatherson 2001: 373)

Each of these is independent of accompaniment yet not, intuitively, intrinsic. If our definition succeeds, for each we should be able to find some possible case in which an individual has the property by virtue of having some other property that is not independent of accompaniment.

Such cases are not hard to find. E2 could be instantiated by an individual by virtue of its having the property of being a lonely round thing. E3 could be instantiated by virtue of an individual's having the property of being one of exactly 5 cubes. In both cases the grounding property is not independent of accompaniment.

Consider another example drawn from that symposium:

(E4) Being a rock (Sider 2001: 361)

Intuitively, E4 is not intrinsic, since it is, as Sider points out, a "bordersensitive" property: whether something counts as a rock depends on what surrounds it. A chunk of appropriate material can be a rock when its border contrasts with its environment-as when a significant portion of it is bordered with air-and it can be a non-rock when it is seamlessly embedded in a larger chunk of the same material. E4 is, notably, independent of accompaniment.

Consider a world in which an individual a is an irregularly shaped chunk of sandstone lying in a field of grass. In this case, in virtue of what property might we say a has E4? If we were to say a has E4 in virtue of having the property of being a chunk of sandstone lying in a field of grass, that would give us the result we want, since nothing can have that property while being lonely. But it is hardly intuitively compelling to say that a has E4 in virtue of having that property. We think what we should say here is, instead, something like this: a has $\mathrm{E} 4$ in virtue of being a chunk of sandstone bordered by something dissimilar to sandstone. This property, of course, is not independent of accompaniment: to have it, one needs to be bordered by some distinct individual.

Why should we accept this "in virtue of" claim? Recall the Joint Sufficiency Principle. In light of this principle, we note that we cannot say that the only property in virtue of which $\mathbf{a}$ is a rock is the property of being a 
chunk of sandstone, since that property is not sufficient for being a rock. The very reason Sider introduced E4 as a case to consider is the fact that something could be made of such material yet not be a rock, as it could be seamlessly embedded in a larger chunk of rock-like material. So there must be something else, in addition to being a chunk of sandstone, in virtue of which a is a rock. Given Sider's observation, it seems a reasonable conjecture that we need to add as one of the grounding properties in this case the property of being bordered by something dissimilar to sandstone. As a result, the same considerations that lead us to consider E4 as not intrinsic lead us to a grounding property that fails to be independent of accompaniment.

These examples should help illustrate the way the definition is meant to work. Are there any clear counterexamples? We'll presume for the sake of simplicity that any counterexamples can be understood as showing that our definition of "has P in an intrinsic fashion" is inadequate. There are, of course, two classes of potential counterexamples: those that challenge the necessity of the conditions set out in that definition and those that challenge their sufficiency. It seems to us that a relatively simple argument can be given to show that there are no counterexamples of the first sort, and we give it below in $\$ 4.2$. No such shortcut is available for evaluating the availability of counterexamples to sufficiency; we'll consider such counterexamples in $\$ 4.3$.

\subsection{Counterexamples to necessity?}

As we noted above, we don't believe that an assessment of the Strong Independence Definition relies solely on particular intuitions about "in virtue of" claims; some general principles-such as the Joint Sufficiency Principle-may be available. We here want to invoke another general "in virtue of" principle which, if correct, makes it plain that no possible counterexample to necessity can be intuitively compelling.

The principle in question is one we already encountered on the way towards motivating our proposed definition. Recall that in $\$ 2.1$ we suggested the following account of why the property of being either cubical and lonely or noncubical and accompanied seemed so obviously not to be intrinsic. It is immediately evident that one way an individual could have that property is in virtue of having the property of being cubical and accompanied, and this latter property is obviously not intrinsic. It is, we suggested, because this fact about the property leaps out at us that we are firmly inclined not to count it as intrinsic.

This diagnosis strongly suggests a generalization about any properties $P$ and $Q$ : if an individual has a property $P$ in virtue of having $Q$, where $Q$ is not intrinsic, then $P$ is not intrinsic either. Note that this generalization is happily congruent with one of the usual orienting characterizations: an intrinsic 
property is one which things have in virtue of the way they themselves are. It is plain that this means: an intrinsic property is one which things have in virtue of the way they themselves intrinsically are. So our generalization seems quite secure.

Now if we modify the generalization only slightly by switching focus to the relativized notion of having a property in an intrinsic fashion, we get the following Intrinsicality Defeater Principle:

(IDP) For any individual $x$ and property $P$, if $x$ has $P$ in virtue of some property that is not itself had in an intrinsic fashion, then $x$ does not have $P$ in an intrinsic fashion.

In light of the usual orienting characterizations and the intuitive power of our diagnosis, we think IDP is extremely plausible.

If we are granted in addition to IDP one further principle which we think all will readily accept, we can show that there are no counterexamples to the necessity of our conditions. The further principle doesn't use "in virtue of" at all but merely states a necessary condition on being had in an intrinsic fashion:

For any individual $x$ and property $P$, if $x$ has $P$ in an intrinsic fashion, $P$ is independent of accompaniment.

Now let us turn to see how these principles can be used to show how counterexamples to the necessity of our definition of "has $P$ in an intrinsic fashion" can be ruled out. A counterexample to the necessity of those conditions would have to be of at least one of the following two sorts:

(A) An individual $x$ has $P$ in an intrinsic fashion yet $P$ is not independent of accompaniment.

(B) An individual $x$ has $P$ in an intrinsic fashion yet there is a property $Q$ such that $x$ has $P$ in virtue of having $Q$ and $Q$ is not independent of accompaniment.

Counterexamples of sort $A$ are easily ruled out. If $P$ is not independent of accompaniment, then it is plainly not had in an intrinsic fashion. Counterexamples of sort $B$ are ruled out nearly as easily. If the property $Q$ is itself not independent of accompaniment, then it is not had in an intrinsic fashion. If it is not had in an intrinsic fashion, then, by IDP, $P$ is not had in an intrinsic fashion either. So B-type cases are also impossible, and there can be no counterexamples to the necessity of our conditions.

We may illustrate the import of this result by looking at an example that has been suggested to us as a problem case: 
Consider the following "in virtue of" claim: one could be alive by virtue of being human. Suppose this is correct. If so, then there is an argument for saying that E5 could be had by virtue of a property that is not independent of accompaniment, since being human arguably requires having a certain historical connection to the race of humans. If so, then, one may put forward E5 as a counterexample to our definition, arguing that E5 is intrinsic, yet it could be had by virtue of a property (being human) which is not independent of accompaniment.

One reaction to such an example is to attempt to assess the particular "in virtue of' claim; is it in fact true that one could be alive in virtue of being human? We are tempted to argue that this cannot be right, since it is more plausible to say that one is human in virtue of being alive (among other things). But we need not resolve the matter. If you are convinced both that being human depends on historical properties and that one can be alive by virtue of being human, we suspect that you will then and there be convinced that being alive is not, after all, an intrinsic property. In other words, the potential counterexample would have shown not that the Strong Independence Definition misclassifies E5; rather, what it would have shown is that our initial classification of E5 as intrinsic was mistaken. ${ }^{7}$ It seems to us that the Intrinsicality Defeater Principle is so firmly endorsed by intuition that any potential counterexample to necessity can be turned aside.

\subsection{Counterexamples to sufficiency?}

Let's turn now to potential counterexamples to sufficiency. These would be cases in which an individual $x$ has $P, P$ is independent of accompaniment, all the "grounding" properties for $x$ 's having $P$ are also independent of accompaniment-yet $x$ does not have $P$ in an intrinsic fashion.

Consider two examples drawn from the symposium on the Naturalness Definition:

(E6) Being such that there is something to which one is attending (Hawthorne 2001: 399)

(E7) Being such that there is a cube (Marshall and Parsons 2001: 349)

7 We note that the following explanation of the mistake might be available. Suppose that while $P$ can be had in a non-intrinsic fashion, as just demonstrated by the example, it can also be had in many cases in an intrinsic fashion; such cases might explain a hasty judgement that $P$ is itself an intrinsic property. 
Both of these are properties which are independent of accompaniment yet not, so far as intuition goes, intrinsic. If our definition succeeds, for each we should be able to find some possible case in which an individual has the property by virtue of having some other property that is not independent of accompaniment. It seems at first relatively easy to find such cases. For the first, we can point to the possibility of something having E6 by virtue of attending to some individual wholly distinct from itself. ${ }^{8}$ For the second, an individual that is not a cube might have $\mathrm{E} 7$ by virtue of being accompanied by a wholly distinct individual that is a cube.

Both claims might be challenged, however. Consider the kind of case we have in mind for E6: let $\mathbf{a}$ and $\mathbf{b}$ be two wholly distinct individuals such that $\mathbf{a}$ is attending to $\mathbf{b}$ but not to herself. Plainly, such a case is possible, but the question is whether we should say that a has E6 by virtue of having this specific property: attending to some wholly distinct individual. Why not (an objector might ask) just say that a has E6 simply by virtue of having the property of attending to something? Why must the grounding property include the "wholly distinct" element?

A parallel question can be asked about the case we invoked for dealing with E7. Suppose $\mathbf{c}$ and $\mathbf{d}$ are two wholly distinct individuals in the same world, where $\mathbf{c}$ is a sphere and $\mathbf{d}$ is a cube. We want to say that $\mathbf{c}$ has E7 by virtue of having this property: being accompanied by a wholly distinct cube. But again, one might press the question: why not just say that $\mathrm{c}$ has $\mathrm{E} 7$ by virtue of having the property of being accompanied by a cube-regardless of whether that cube is wholly distinct or not?

The first thing we want to note in addressing this worry is that we can accept multiple, overlapping grounding properties. We are not forced to choose between saying that $\mathbf{c}$ has $\mathrm{E} 7$ by virtue of being accompanied by a cube and saying that $\mathbf{c}$ has E7 by virtue of being accompanied by a wholly distinct cube. Both can be true, and all our definition requires is that at least the second of them be true.

Still, that point made, one may wonder whether there is any positive reason to include the needed property - the one that fails to be independent of accompaniment-as one in virtue of which the target property is had. We think there is. Let's consider the case of $\mathbf{c}, \mathbf{d}$, and $\mathrm{E} 7$ first. Note that both $\mathbf{c}$ and $\mathbf{d}$ have $\mathrm{E} 7$. Question: do $\mathrm{c}$ and $\mathrm{d}$ have $\mathrm{E} 7$ in the same way, or is there

8 The following objection to our treatment of this case has been suggested to us. Suppose a is attending to a wholly distinct individual $b$. Then a has $\mathrm{E} 6$ in virtue of the property of attending to someone wholly distinct from himself-or so we would like to say. But might we not better say that a has E6 in virtue of the (impure) property of attending to $\mathbf{b}$ ? That property, interestingly enough, is independent of accompaniment (b could have it while being lonely). In reply, we point out that to the degree that it is plausible to suppose that a has E6 in virtue of having the property of attending to $\mathbf{b}$, it is just as plausible to suppose that a has E6 in virtue of the property being $a$ and attending to $b$. Aind this latter property is not independent of accompaniment. 
something importantly different about the way in which each has this property? It seems intuitively compelling to say that the way in which $c$ has E7 is quite different from the way in which $d$ has E7. After all, $d$ has E7 by-we want to say-simply being, itself, a cube, whereas $\mathbf{c}$ has the same property in a very different way-by being accompanied by something quite different from itself that happens to be a cube.

If we are right that the way $\mathbf{c}$ has $\mathrm{E} 7$ differs from the way $\mathbf{d}$ has $\mathrm{E} 7$, then the question arises: what is the nature of that difference? A plausible answer locates the difference in different grounding properties: there is some property $P$ such that $c$ has $E 7$ by virtue of having $P$ and $d$ does not have $E 7$ by virtue of having $P$. A plausible candidate for $P$ is, we think, the property of being accompanied a wholly distinct cube.

We think something similar should be said about E6. Consider $\mathbf{a}$ and $\mathbf{b}$ again; let's add, this time, that while $\mathbf{a}$ attends to $\mathbf{b}$ but not herself, $\mathbf{b}$ attends to himself but not to a. In this case, shouldn't we say that a has E6 in a different way from the way $\mathbf{b}$ has E6? If so, we need to account for that difference; to account for the difference we need to find distinct grounding properties, and a plausible story about the difference is that a has E6 by virtue of attending to some wholly distinct individual, whereas $\mathbf{b}$ has E6 by virtue of attending to himself.

Our examination of these cases suggests another general heuristic for helping stabilize "in virtue of" judgements. When considering an individual's possession of a property $P$, we might ask whether there is any distinct $Q$ such that $P$ is had by that individual by virtue of having $Q$; we can pursue this question by considering other possible individuals which have $P$ and asking whether it is intuitively compelling to say that those have $\mathrm{P}$ in a different way than the original individual.

We can hardly pretend to have exhausted all possible counterexamples to the sufficiency of our definition, of course, but we hope that this short survey demonstrates the power and promise of that definition.

\subsection{Two special categories}

Before closing, we want to address two special categories of property that seem to cause trouble for the Strong Independence Definition. We'll argue, however, that these properties may be safely neglected, so that it doesn't matter how our definition classifies them.

The first category is that of what we call the indiscriminately essential properties. A property $P$ is indiscriminately essential just in case every individual-both possible and actual-has it necessarily. If we presume numbers exist necessarily, the property of being accompanied by the number 21 is such a property. The point to notice here is that if a property is indiscriminately essential, it cannot be classified as intrinsic according to our definition. 
That definition requires of any intrinsic property that it be independent of accompaniment. For a property to be independent of accompaniment, it must be possible for a lonely individual to lack it and for an accompanied individual to lack it; but of course no individual of any sort could lack an indiscriminately essential property. So our definition classifies every such property as not intrinsic.

This may seem all well and good for such properties as that of being accompanied by the number 21 . But what of $E 8$ ?

(E8) Being self-identical

Plainly, E8 is indiscriminately essential. So we are forced to say it is not intrinsic. But it is not clear that this is the right classification. After all, an individual's having E8 does not depend on anything outside of that individual. Is this then a counterexample to the Strong Independence Definition?

The situation is a bit bewildering, and we think we know why. The usual orienting characterizations of intrinsicality often focus on a negative point: an intrinsic property is one that doesn't depend on anything other than the way that individual itself is. Certainly the property of being self-identical doesn't depend on anything outside the individual in question. The problem is that it doesn't depend on anything inside the individual either-it is simply independent of everything. What we want to suggest is that the notion of intrinsicality is intended only to draw a distinction among properties that depend on how things are contingently: there are the properties that depend only on how the thing itself is, and then there are the properties that depend also (and perhaps only) on how other things are. Properties that don't depend on how anything is at all can be safely set aside as irrelevant. The upshot is this: we think our definition of intrinsicality need only capture the intuitively correct extension among properties that are not indiscriminately essential.

The second category of properties that needs special treatment is the category of impure properties. An impure property is one the expression of which makes ineliminable reference to particular individuals, such as the property of being a student of Quine. ${ }^{9}$ Now consider this impure property:

(E9) Being identical with George Washington

Let us ask two questions about E9. First, does our Strong Independence Definition count it as intrinsic? Second, does intuition count it as intrinsic?

9 There is a good question-which we don't want to tackle here-about how, exactly, we should distinguish this class of properties. What we've used in the main text is a linguistic criterion: a property the expression of which makes ineliminable reference to a particular individual. Some non-linguistic criterion would be preferable, but we don't have one. 
As to the first, the answer is Yes. The property is independent of accompaniment, as it is possible for Washington to exist alone, for him to exist accompanied, for someone else to exist alone and for someone else to exist accompanied. Is it possible for it to be instantiated by virtue of Washington's having some other property that isn't independent of accompaniment? We don't think so. As curious a property as this is, one thing we think we know about it is that it can never be instantiated by virtue of a distinct property. So our definition seems to count it as intrinsic.

But should we count it as intrinsic? As Theodore Sider (1995: 4) has pointed out, one can argue to the contrary on the following grounds. We have the notion of two things being perfect intrinsic duplicates of each other. Obviously, if two things are perfect intrinsic duplicates of each other, there is no intrinsic property with regards to which they differ. But if we count the property of being identical with George Washington as intrinsic, we face a problem, for it seems intuitively correct that there could be a George Washington twin-that is, an individual, distinct from George Washington, who is nonetheless a perfect intrinsic duplicate of George Washington. If we count the property of being identical with George Washington as intrinsic, such a situation is impossible. So there is some intuitive force to saying that E9 is not, after all, intrinsic.

But intuition is not in fact so clear. After all, does Washington's possession of this property depend on anything outside of himself? It is not, like the property of being self-identical, entirely independent of contingencies. So why not classify it as intrinsic? When George Washington has this property, isn't it something he possesses solely by virtue of who he, himself, is? It doesn't seem to be, by contrast, a relational property: being George Washington isn't in any sense a property one has by virtue of being related to some distinct individual.

The proper response here, we think, is similar to that which we gave above for the indiscriminately essential properties. The notion of intrinsicality with which we are concerned is one meant to draw a distinction among the pure properties; for our definition to succeed, it need only capture the intuitively correct extension among those properties that are neither impure nor indiscriminately essential. The fact that intuition delivers no clear verdict on this point argues in favor of this restriction..$^{10,11}$

10 We note that our claim is not that "in virtue of" fails to apply in such cases. Plausibly, the impure property of being a citizen of the United States can be possessed by virtue of being bom in the United States. Our claim is only that the classification of properties as intrinsic or not is not meant to apply to such cases.

11 We'd like to thank two anonymous referees for PPR for their extensive and useful comments on an earlier version of this paper. Thanks are also due to the participants at the Florida Philosophical Association conference in November 2002, where a brief version of this paper was presented. Finally, we want to acknowledge the generous support pro- 


\section{REFERENCES}

When reference is made to a reprint, all pagination refers to that in the reprint.

Dunn, J. M. 1990. "Relevant Predication 2: Intrinsic Properties and Internal Relations," Philosophical Studies 60: 177-206.

Francescotti, R. 1999. "How to Define Intrinsic Properties," Nô̂s 33: 590609.

Hawthorne, J. 2001. "Intrinsic Properties and Natural Relations," Philosophy and Phenomenological Research 63(2): 399-403.

Humberstone, I. L. 1996. "Intrinsic/Extrinsic," Synthese 108: 205-267.

Langton, R. and Lewis, D. 1998. "Defining 'Intrinsic'," Philosophy and Phenomenological Research 58, 333-45. Reprinted (1999) in Lewis' Papers in Metaphysics and Epistemology (Cambridge: Cambridge University Press).

Langton, R. and Lewis, D. 2001. "Marshall and Parsons on 'Intrinsic'," Philosophy and Phenomenological Research 63(2): 353-355.

Lewis, D. 1983. "New Work for a Theory of Universals," Australasian Journal of Philosophy 61, pp. 343-377. Reprinted (1999) in his Papers in Metaphysics and Epistemology (Cambridge: Cambridge University Press).

Lewis, D. 1986. On the Plurality of Worlds. New York: Blackwell.

Lewis, D. 2001. "Redefining 'Intrinsic'," Philosophy and Phenomenological Research 63(2): 381-398.

Marshall, D. and Parsons, J. 2001. 'Langton and Lewis on 'Intrinsic'," Philosophy and Phenomenological Research 63(2): 347-351.

Sider, T. 1995. "Intrinsic Properties," Philosophical Studies 83: 1-27.

Sider, T. 2001. "Maximality and Intrinsic Properties," Philosophy and Phenomenological Research 63(2): 357-363.

Taylor, B. 1993. "On Natural Properties in Metaphysics," Mind 102: 83-100.

Vallentyne, P. 1997. "Intrinsic Properties Defined," Philosophical Studies 88: 209-219.

Weatherson, B. 2001. "Intrinsic Properties and Combinatorial Principles," Philosophy and Phenomenological Research 63(2): 365-380.

Zimmerman, D. 1997. "Immanent Causation." In J. Tomberlin, ed., Philosophical Perspectives 11: Mind, Causation and World: 433-471. Cambridge: Blackwell.

vided by a Humanities Scholarship Enhancement Fund awarded to Gene Witmer in the summer of 2002 by the College of Liberal Arts and Sciences. 